\title{
Small Bowel Evaluation in Asymptomatic Fecal Immunochemical Test-Positive Patients with a Negative Colonoscopy: Is It Necessary?
}

\author{
Jae Jun Park · Jae Hee Cheon
}

Published online: 29 July 2011

(C) Springer Science+Business Media, LLC 2011

Screening for colorectal cancer (CRC) and its precursors by fecal occult blood test (FOBT) is widely recommended and is being applied more than ever [1-3]. Although annual or biennial guaiac fecal occult blood test (gFOBT) screening has been proved to reduce the mortality of CRC [4], the gFOBT has been faulted for its low sensitivity $[5,6]$. More recently, the fecal immunochemical test (FIT) has been introduced as an alternative to the gFOBT. The FIT is currently preferred over the gFOBT due to its higher sensitivity and improved detection rate for advanced neoplasia [5, 7-10]. Besides its diagnostic performance, the FIT has several advantages over the gFOBT including no need for dietary restriction, easier stool collection method, and automated analysis [11]. Furthermore, quantitative FIT allows for the adjustment of the cutoff value for an abnormal result based on population characteristics and available colonoscopy resources. Due to these characteristics, quantitative FIT is more favored as a screening tool than qualitative FIT [11].

The FIT selectively reacts with the globin moiety of human hemoglobin [12]. Because globin is rapidly degraded by proteases in the upper gastrointestinal (GI) tract, the FIT dose not detect small amounts of blood in the upper GI tract and selectively recognizes occult bleeding of colorectal origin [12]. With regard to small bowel lesions,

J. J. Park

Department of Internal Medicine, Kyung Hee University

Hospital at Gangdong, Kyung Hee University, Seoul, Korea

\section{J. H. Cheon $(\square)$}

Department of Internal Medicine and Institute

of Gastroenterology, Yonsei University College of Medicine,

50 Yonsei-ro, Seodaemun-gu, Seoul 120-752, Korea

e-mail: geniushee@yuhs.ac although there is a possibility that some small bowel occult bleeding could be identified by FIT, no data are currently available regarding this issue.

In the current issue of Digestive Diseases and Sciences, Chiba et al. [13] present the results of their prospective study on small bowel evaluation in asymptomatic FIT-positive subjects with a negative colonoscopy. In this, the first study to evaluate the small bowel with capsule endoscopy (CE) in asymptomatic FIT-positive patients with a negative colonoscopy, 53 asymptomatic patients with FIT-positivity and a negative total colonoscopy and esophagogastroduodenoscopy underwent CE. The CE findings were classified into three categories: $\mathrm{P} 0$, no abnormalities or findings without potential for bleeding; P1, findings with uncertain potential for bleeding; and P2, findings with high potential for bleeding. The cecal completion rate was $92.5 \%$. There were no cases of $\mathrm{P} 2,19$ cases of $\mathrm{P} 1$, and 34 cases of $\mathrm{P} 0$. Moreover, no abnormalities were identified in five patients who underwent additional diagnostic evaluations that were considered necessary based on the individual clinical results. No clinically significant small bowel lesions were found in the asymptomatic FIT-positive patients with negative bidirectional endoscopy.

Although FIT has been widely accepted as a CRC screening tool, $40-60 \%$ of subjects with a positive FIT are found to have no lesions in their colon or rectum to explain their positive status $[5,14,15]$. How then do we explain the discrepancy between the results of FIT and colonoscopy? There are several possible explanations for this discrepancy. First, this may reflect true false-positive results of FIT for advanced neoplasia. Quantitative FIT results are subjected to predetermined cut-off values for fecal hemoglobin; this methodology could inevitably yield some true false positives. Additionally, medications that can increase the likelihood of GI bleeding may cause false-positive FIT 
results. However, recent data, although not sufficient, demonstrated that low-dose aspirin, nonsteroidal antiinflammatory drugs (NSAIDS), and anticoagulant use have no or marginal effects on the specificity of FIT, rather they increase the sensitivity of FIT $[16,17]$. At this time, concomitant use of those drugs does not seem to be the culprit behind FIT false-positive results. More studies are needed to elucidate this issue. Beyond this, sample contamination by menstruation may also lead to false-positive FIT results. Second, there may be significant lesions that are missed during endoscopic examinations. According to tandem colonoscopy data, the adenoma miss rate of lesions $\geq 10 \mathrm{~mm}$ size is $1-8 \%$ [18]. Moreover, in cases of suboptimal bowel preparation, the miss rate increases considerably, up to $36 \%$ [19]. With this in mind, clinicians should verify the quality of the colonoscopy when they are faced with unexplained colonoscopy results in FIT-positive subjects. Additionally, significant small bowel lesions may be missed by CE. The potential miss rate of $\mathrm{CE}$ has been reported to be approximately $20 \%$ [20]. The observation that the rebleeding rate for patients with obscure GI bleeding and a negative $\mathrm{CE}$ is considerable also reflects this significant miss rate [21]. Third, FIT may yield positive results for small adenomas. Morikawa et al. [22] reported that FIT detects small adenomas $(\leq 9 \mathrm{~mm})$ in men with a level of sensitivity significantly higher than serendipity, although the sensitivity is low. In contrast to asymptomatic subjects, it is noteworthy that, in cases of overt obscure GI bleeding or iron deficiency anemia, a substantial portion of cases had significant small bowel lesions, as demonstrated by data presented by Chiba et al. [13] and shown in their Table 2.

In clinical practice, patients with a false-positive FIT can embarrass physicians. Is additional small bowel exploration needed? What kind of clinical management should be provided? As shown by the work of Chiba et al. [13], the role of CE is limited in asymptomatic patients with a positive FIT; these findings partly relieve concern for the need for small bowel evaluation in these patients. At present, a regular follow-up with repeated FITs and consideration of repeated or earlier follow-up colonoscopy according to the quality of the initial endoscopy may be an appropriate management strategy.

In order to determine the sensitivity of FIT for detecting any type of small bowel bleeding, additional studies of FIT in patients who are undergoing CE or deep enteroscopy examination for suspected small bowel lesions are needed. These future studies may reveal the true performance of FIT in detecting small bowel lesions with respect to the detectable degree of bleeding and the exact locations of these lesions in the small bowel. Moreover, long-term follow-up studies for FIT positive patients with a nonexplanatory colonoscopy are also warranted.
In summary, additional small bowel evaluation is not recommended in asymptomatic FIT-positive patients with a negative colonoscopy. However, further small bowel evaluation should be considered in patients with clinical symptoms, iron deficiency anemia, or gross bleeding after negative conventional endoscopic examinations.

\section{References}

1. Rex DK, Johnson DA, Anderson JC, et al. American College of Gastroenterology guidelines for colorectal cancer screening 2009 [corrected]. Am J Gastroenterol. 2009;104:739-750.

2. Levin B, Lieberman DA, McFarland B, et al. Screening and surveillance for the early detection of colorectal cancer and adenomatous polyps, 2008: a joint guideline from the American Cancer Society, the US Multi-Society Task Force on Colorectal Cancer, and the American College of Radiology. Gastroenterology. 2008;134:1570-1595.

3. Stock C, Brenner H. Utilization of lower gastrointestinal endoscopy and fecal occult blood test in 11 European countries: evidence from the Survey of Health, Aging and Retirement in Europe (SHARE). Endoscopy. 2010;42:546-556.

4. Hewitson P, Glasziou P, Watson E, et al. Cochrane systematic review of colorectal cancer screening using the fecal occult blood test (hemoccult): an update. Am J Gastroenterol. 2008;103: 1541-1549.

5. van Rossum LG, van Rijn AF, Laheij RJ, et al. Random comparison of guaiac and immunochemical fecal occult blood tests for colorectal cancer in a screening population. Gastroenterology. 2008;135:82-90.

6. Imperiale TF, Ransohoff DF, Itzkowitz SH, et al. Fecal DNA versus fecal occult blood for colorectal-cancer screening in an average-risk population. $N$ Engl J Med. 2004;351:2704-2714.

7. Guittet L, Bouvier V, Mariotte N, et al. Comparison of a guaiac based and an immunochemical faecal occult blood test in screening for colorectal cancer in a general average risk population. Gut. 2007;56:210-214.

8. Allison JE, Sakoda LC, Levin TR, et al. Screening for colorectal neoplasms with new fecal occult blood tests: update on performance characteristics. J Natl Cancer Inst. 2007;99:1462-1470.

9. Hundt S, Haug U, Brenner H. Comparative evaluation of immunochemical fecal occult blood tests for colorectal adenoma detection. Ann Intern Med. 2009;150:162-169.

10. Lieberman D. Colon cancer screening and surveillance controversies. Curr Opin Gastroenterol. 2009;25:422-427.

11. Duffy MJ, van Rossum LG, van Turenhout ST, et al. Use of faecal markers in screening for colorectal neoplasia: a European group on tumor markers position paper. Int J Cancer. 2011;128: 3-11.

12. Rockey DC. Occult gastrointestinal bleeding. $N$ Engl J Med. 1999;341:38-46.

13. Chiba H, Sekiguchi M, Ito T, et al. Is it worthwhile to perform capsule endoscopy for asymptomatic patients with positive immunochemical faecal occult blood test? Dig Dis Sci. (Epub ahead of print). doi:10.1007/s10620-011-1798-4.

14. Levi Z, Rozen P, Hazazi R, et al. A quantitative immunochemical fecal occult blood test for colorectal neoplasia. Ann Intern Med. 2007; 146:244-255.

15. Park DI, Ryu S, Kim YH, et al. Comparison of guaiac-based and quantitative immunochemical fecal occult blood testing in a population at average risk undergoing colorectal cancer screening. Am J Gastroenterol. 2010;105:2017-2025. 
16. Levi Z, Rozen P, Hazazi R, et al. Sensitivity, but not specificity, of a quantitative immunochemical fecal occult blood test for neoplasia is slightly increased by the use of low-dose aspirin, NSAIDs, and anticoagulants. Am J Gastroenterol. 2009;104: 933-938.

17. Brenner H, Tao S, Haug U. Low-dose aspirin use and performance of immunochemical fecal occult blood tests. JAMA. 2010;304:2513-2520.

18. van Rijn JC, Reitsma JB, Stoker J, et al. Polyp miss rate determined by tandem colonoscopy: a systematic review. Am J Gastroenterol. 2006;101:343-350.

19. Lebwohl B, Kastrinos F, Glick M, et al. The impact of suboptimal bowel preparation on adenoma miss rates and the factors associated with early repeat colonoscopy. Gastrointest Endosc. 2011;73:1207-1214.

20. Mehdizadeh S, Ross A, Gerson L, et al. What is the learning curve associated with double-balloon enteroscopy? Technical details and early experience in 6 U.S. tertiary care centers. Gastrointest Endosc. 2006;64:740-750.

21. Park JJ, Cheon JH, Kim HM, et al. Negative capsule endoscopy without subsequent enteroscopy does not predict lower long-term rebleeding rates in patients with obscure GI bleeding. Gastrointest Endosc. 2010;71:990-997.

22. Morikawa T, Kato J, Yamaji Y, et al. Sensitivity of immunochemical fecal occult blood test to small colorectal adenomas. $\mathrm{Am}$ J Gastroenterol. 2007;102:2259-2264. 